\title{
GLOBAL ASYMPTOTIC STABILITY OF SOLUTIONS OF CUBIC STOCHASTIC DIFFERENCE EQUATIONS
}

\author{
ALEXANDRA RODKINA AND HENRI SCHURZ
}

Received 18 September 2003 and in revised form 22 December 2003

Global almost sure asymptotic stability of solutions of some nonlinear stochastic difference equations with cubic-type main part in their drift and diffusive part driven by square-integrable martingale differences is proven under appropriate conditions in $\mathbb{R}^{1}$. As an application of this result, the asymptotic stability of stochastic numerical methods, such as partially drift-implicit $\theta$-methods with variable step sizes for ordinary stochastic differential equations driven by standard Wiener processes, is discussed.

\section{Introduction}

Suppose that a filtered probability space $\left(\Omega, \mathscr{F}_{n},\left\{\mathscr{F}_{n}\right\}_{n \in \mathbb{N}}, \mathbb{P}\right)$ is given as a stochastic basis with filtrations $\left\{\mathscr{F}_{n}\right\}_{n \in \mathbb{N}}$. Let $\left\{\xi_{n}\right\}_{n \in \mathbb{N}}$ be a one-dimensional real-valued $\left\{\mathscr{F}_{n}\right\}_{n \in \mathbb{N}}$ martingale difference (for details, see $[2,14]$ ) and let $\mathscr{B}(S)$ denote the set of all Borel sets of the set $S$. Furthermore, let $a=\left\{a_{n}\right\}_{n \in \mathbb{N}}$ be a nonincreasing sequence of strictly positive real numbers $a_{n}$ and let $\kappa=\left\{\kappa_{n}\right\}_{n \in \mathbb{N}}$ be a sequence of real numbers $\kappa_{n}$. We use "a.s." as the abbreviation for wordings " $\mathbb{P}$-almost sure" or " $\mathbb{P}$-almost surely".

In this paper, we consider discrete-time stochastic difference equations (DSDEs)

$$
x_{n+1}-x_{n}=\kappa_{n} x_{n}^{3}-a_{n} x_{n+1}^{3}+f_{n}\left(\left(x_{l}\right)_{0 \leq l \leq n}\right)+\sigma_{n}\left(\left(x_{l}\right)_{0 \leq l \leq n}\right) \xi_{n+1}
$$

with cubic-type main part of their drift in $\mathbb{R}^{1}$, real parameters $a_{n}, \kappa_{n} \in \mathbb{R}^{1}$, driven by the square-integrable martingale difference $\xi=\left\{\xi_{n+1}\right\}_{n \in \mathbb{N}}$ of independent random variables $\xi_{n+1}$ with $\mathbb{E}\left[\xi_{n+1}\right]=0$ and $\mathbb{E}\left[\xi_{n+1}\right]^{2}<+\infty$. We are especially interested in conditions ensuring the almost sure global asymptotic stability of solutions of these DSDEs (1.1). The main result should be such that it can be applied to numerical methods for related continuous-time stochastic differential equations (CSDEs) as its potential limits. For example, consider

$$
d X_{t}=\left(a^{1}\left(t, X_{t}\right)+a^{2}\left(t, X_{t}\right)\right) d t+b\left(t, X_{t}\right) d W_{t}
$$


driven by standard Wiener process $W=\left\{W_{t}\right\}_{t \geq 0}$ and interpreted in the Itô sense, where $a^{1}, a^{2}, b:[0,+\infty) \times \mathbb{R} \rightarrow \mathbb{R}$ are smooth vector fields. Such CSDEs (1.2) with additive drift splitting can be discretized in many ways; for example, see [13] for an overview. However, only few of those discretization methods are appropriate to tackle the problem of almost sure asymptotic stability of their trivial solutions. One of the successful classes is that of partially drift-implicit $\theta$-methods with the schemes

$$
\begin{aligned}
x_{n+1}= & x_{n}+\left(\theta_{n} a^{1}\left(t_{n+1}, x_{n+1}\right)+\left(1-\theta_{n}\right) a^{1}\left(t_{n}, x_{n}\right)+a^{2}\left(t_{n}, x_{n}\right)\right) \Delta_{n} \\
& +b\left(t_{n}, x_{n}\right) \Delta W_{n}
\end{aligned}
$$

applied to equation (1.2), where $\Delta_{n}=t_{n+1}-t_{n}$ and $\Delta W_{n}=W_{t_{n+1}}-W_{t_{n}}$, along any discretizations $0=t_{0} \leq t_{1} \leq \cdots \leq t_{N}=T$ of time intervals $[0, T]$. These methods with uniformly bounded $\theta_{n}$ (with $\sup _{n \in \mathbb{N}}\left|\theta_{n}\right|<+\infty$ ) provide $L^{2}$-converging approximations to (1.2) with rate 0.5 in the worst case under appropriate conditions on $a^{1}, a^{2}, b$. For details, see $[8,10,13]$. Obviously, schemes (1.3) applied to Itô-type CSDEs

$$
d X_{t}=\left(f\left(t, X_{t}\right)-\gamma^{2}\left[X_{t}\right]^{3}\right) d t+b\left(t, X_{t}\right) d W_{t}
$$

possess the form of $(1.1)$ with $a_{n}=\theta_{n} \gamma^{2} \Delta_{n}, \kappa_{n}=\left(\theta_{n}-1\right) \gamma^{2} \Delta_{n}, f_{n}\left(\left(x_{l}\right)_{0 \leq l \leq n}\right)=f\left(t_{n}, x_{n}\right) \Delta_{n}$, $a^{1}(t, x)=-\gamma^{2} x^{3}, a^{2}(t, x)=f(t, x), \sigma_{n}\left(\left(x_{l}\right)_{0 \leq l \leq n}\right)=b\left(t_{n}, x_{n}\right)$, and $\Delta W_{n}=\xi_{n+1}$. Thus, assertions on the asymptotic behavior of (1.1) help us to understand the asymptotic behavior of methods (1.3) and provide criteria to choose possibly variable step sizes $\Delta_{n}$ in its algorithm such that asymptotic stability can be guaranteed for the discretization of the related continuous-time system too. In passing, we note that, in the bilinear case, moment stability issues have been examined for corresponding drift-implicit $\theta$ - and trapezoidal methods in $[8,9,11,12,13]$. Here, we concentrate on almost sure stability issues of nonlinear and nonautonomous subclasses of (1.1) exclusively, in particular, when discretized by additive drift splitting methods with variable step sizes $\Delta_{n}$. Effects of nonlinearities on the stability behavior of discrete integrodifference equations subjected to bounded perturbations and cubic terms are studied in $[1,4,7]$ by using Lyapunov functionals.

\section{Auxiliary statements}

The following lemma is a generalization of Doob decomposition of submartingales (for details, see $[2,14])$.

Lemma 2.1. Let $\left\{\xi_{n}\right\}_{n \in \mathbb{N}}$ be an $\left\{\mathscr{F}_{n}\right\}_{n \in \mathbb{N}}$-martingale difference. Then there exist an $\left\{\mathscr{F}_{n}\right\}_{n \in \mathbb{N}}$-martingale difference $\mu=\left\{\mu_{n}\right\}_{n \in \mathbb{N}}$ and a positive $\left(\mathscr{F}_{n-1}, \mathscr{B}_{B}\left(\mathbb{R}^{1}\right)\right)$-measurable (i.e., predictable) stochastic sequence $\eta=\left\{\eta_{n}\right\}_{n \in \mathbb{N}}$ such that, for every $n=1,2, \ldots$ a.s.,

$$
\xi_{n}^{2}=\mu_{n}+\eta_{n}
$$

The process $\left\{\eta_{n}\right\}_{n \in \mathbb{N}}$ can be represented by $\eta_{n}=\mathbb{E}\left(\xi_{n}^{2} \mid \mathscr{F}_{n-1}\right)$. Moreover, $\eta=\left(\eta_{n}\right)_{n \in \mathbb{N}}$ is a nonrandom sequence when $\xi_{n}$ are independent random variables. In this case,

$$
\eta_{n}=\mathbb{E}\left(\xi_{n}^{2}\right), \quad \mu_{n}=\xi_{n}^{2}-\mathbb{E}\left(\xi_{n}^{2}\right) .
$$


To establish asymptotic stability, we will also make use of a certain application of wellknown martingale convergence theorems (cf. [14]) in the form of Lemma 2.2 which is originally proved in [15, Lemma A, page 243].

Lemma 2.2. Let $Z=\left\{Z_{n}\right\}_{n \in \mathbb{N}}$ be a nonnegative decomposable stochastic process with DoobMeyer decomposition $Z_{n}=Z_{0}+A_{n}^{1}-A_{n}^{2}+M_{n}$, where $A^{1}=\left\{A_{n}^{1}\right\}_{n \in \mathbb{N}}$ and $A^{2}=\left\{A_{n}^{2}\right\}_{n \in \mathbb{N}}$ are a.s. nondecreasing, predictable processes with $A_{0}^{1}=A_{0}^{2}=0$, and $M=\left\{M_{n}\right\}_{n \in \mathbb{N}}$ is a local

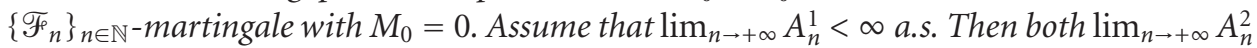
and $\lim _{n \rightarrow+\infty} Z_{n}$ exist and are finite.

Lemma 2.3. For every $a \geq 0$, the function $x \rightarrow F(x)=x+a x^{3}$ is strictly increasing and uniquely invertible with strictly increasing Lipschitz continuous inverse $F^{-1}$ satisfying

$$
\forall y_{1}, y_{2} \in \mathbb{R}^{1}, \quad\left|F^{-1}\left(y_{1}\right)-F^{-1}\left(y_{2}\right)\right| \leq\left|y_{1}-y_{2}\right|
$$

Proof. If $x_{1}<x_{2}$, then there is some intermediate value $\theta \in\left(x_{1}, x_{2}\right)$ (or $\theta \in\left(x_{2}, x_{1}\right)$ if $\left.x_{1} \geq x_{2}\right)$ such that $F\left(x_{1}\right)-F\left(x_{2}\right)=F^{\prime}(\theta)\left(x_{1}-x_{2}\right)=\left(1+3 a \theta^{2}\right)\left(x_{1}-x_{2}\right)<0$, hence $F$ is strictly increasing. Any strictly monotone function is invertible. Therefore, the inverse of $F$ exists and is strictly monotone as well. The strict monotonicity of the inverse $F^{-1}$ is also clear from the mean value theorem. To show (2.3), just note that $F^{\prime}(x) \geq 1$, hence $0 \leq F^{-1 \prime}(x) \leq 1$, and relation (2.3) is apparent. Consequently, the proof is complete.

\section{Almost sure global asymptotic stability of (1.1)}

We suppose that the difference equation (1.1) has nonrandom coefficients satisfying

$$
\forall n \in \mathbb{N}, \quad a_{n}>\left|\kappa_{n}\right|,
$$

with nonincreasing sequence $a=\left\{a_{n}\right\}_{n \in \mathbb{N}}$, and there exist nonnegative nonrandom numbers $\lambda_{n}, \delta_{n}^{(1)}, \delta_{n}^{(2)}, \delta_{n}^{(3)} \in \mathbb{R}_{+}$for all $n \in \mathbb{N}$ such that

$$
\begin{gathered}
\left|\sigma_{n}\left(\left(x_{l}\right)_{0 \leq l \leq n}\right)\right|^{2} \leq \lambda_{n}\left(1+x_{n}^{6}\right)+\delta_{n}^{(1)} x_{n}^{4}+\delta_{n}^{(2)} x_{n}^{6}, \quad \sum_{n=1}^{+\infty} \lambda_{n} \mathbb{E}\left[\xi_{n+1}^{2}\right]<+\infty \\
\left|f_{n}\left(\left(x_{l}\right)_{0 \leq l \leq n}\right)\right|^{2} \leq \delta_{n}^{(3)} x_{n}^{6}
\end{gathered}
$$

Furthermore, we assume that $\delta_{n}^{(j)}, j=1,2,3$, are small enough such that there exist some nonrandom real constants $N_{1} \geq 0, \varepsilon_{1} \geq 0, \varepsilon_{2} \geq 0$ with $\varepsilon_{1}+\varepsilon_{2}>0$ such that for all $n \geq N_{1}$,

$$
\begin{gathered}
2\left(a_{n}-\kappa_{n}\right)-2 \sqrt{\delta_{n}^{(3)}}-\delta_{n}^{(1)} \eta_{n+1} \geq \varepsilon_{1}, \\
\left(a_{n}^{2}-\kappa_{n}^{2}\right)-\delta_{n}^{(2)} \eta_{n+1}-\delta_{n}^{(3)}-2 \kappa_{n} \sqrt{\delta_{n}^{(3)}}-\lambda_{n} \eta_{n+1} \geq \varepsilon_{2} .
\end{gathered}
$$


Theorem 3.1. Let $\xi_{n+1}$ be square-integrable, independent random variables $(n \in \mathbb{N})$ with $\mathbb{E}\left[\xi_{n+1}\right]=0$ and conditions (3.1), (3.2), (3.3), (3.4), and (3.5) be fulfilled. Then the solution $x_{n}$ of equation (1.1) for every initial condition $x_{0}$ has the property that $\lim _{n \rightarrow+\infty} x_{n}=0$ a.s., that is, if additionally $\sigma$ and $f$ have 0 as their trivial equilibrium, then 0 is an asymptotically stable equilibrium with probability one.

Proof. First, note that equation (1.1) can be rewritten equivalently to

$$
\begin{aligned}
F_{n+1}\left(x_{n+1}\right)+\left(a_{n}-a_{n+1}\right) x_{n+1}^{3}= & F_{n}\left(x_{n}\right)-\left(a_{n}-\kappa_{n}\right) x_{n}^{3} \\
& +f_{n}\left(\left(x_{l}\right)_{0 \leq l \leq n}\right)+\sigma_{n}\left(\left(x_{l}\right)_{0 \leq l \leq n}\right) \xi_{n+1},
\end{aligned}
$$

where $F_{n}(x)=x+a_{n} x^{3}$ for $x \in \mathbb{R}^{1}$. We also observe that

$$
\begin{aligned}
F_{n+1}^{2}\left(x_{n+1}\right) & \leq F_{n+1}^{2}\left(x_{n+1}\right)+2\left(a_{n}-a_{n+1}\right) x_{n+1}^{3} F_{n+1}\left(x_{n+1}\right)+\left(a_{n}-a_{n+1}\right)^{2} x_{n+1}^{6} \\
& =\left(F_{n+1}\left(x_{n+1}\right)+\left(a_{n}-a_{n+1}\right) x_{n+1}^{3}\right)^{2}
\end{aligned}
$$

due to the assumption of nonincreasing $\left\{a_{n}\right\}_{n \in \mathbb{N}}$ and the monotone structure of the sequence $\left\{F_{n}(x)\right\}_{n \in \mathbb{N}}$ for any $x \in \mathbb{R}^{1}$. Using Lemma 2.1 and taking the square at both sides of (3.6) lead to

$$
\begin{aligned}
F_{n+1}^{2}\left(x_{n+1}\right) \leq & F_{n}^{2}\left(x_{n}\right)-2\left(a_{n}-\kappa_{n}\right) F_{n}\left(x_{n}\right) x_{n}^{3}+\left(a_{n}-\kappa_{n}\right)^{2} x_{n}^{6} \\
& +2 f_{n}\left(\left(x_{l}\right)_{0 \leq l \leq n}\right)\left(F_{n}\left(x_{n}\right)-\left(a_{n}-\kappa_{n}\right) x_{n}^{3}\right) \\
& +f_{n}^{2}\left(\left(x_{l}\right)_{0 \leq l \leq n}\right)+\sigma_{n}^{2}\left(\left(x_{l}\right)_{0 \leq l \leq n}\right) \eta_{n+1}+\Delta m_{n+1}^{(1)},
\end{aligned}
$$

where $\eta_{n+1}=\mathbb{E}\left[\xi_{n+1}^{2}\right]$, and the therein occurring expression

$$
\Delta m_{n+1}^{(1)}=2\left(F_{n}\left(x_{n}\right)-\left(a_{n}-\kappa_{n}\right) x_{n}^{3}+f_{n}\left(\left(x_{l}\right)_{0 \leq l \leq n}\right)\right) \sigma_{n}(\cdots) \xi_{n+1}+\sigma_{n}^{2}(\cdots) \mu_{n+1}
$$

with $\mu_{n+1}=\xi_{n+1}^{2}-\mathbb{E}\left[\xi_{n+1}^{2}\right]$, is a martingale difference. Note that

$$
\begin{gathered}
F_{n}\left(x_{n}\right)-\left(a_{n}-\kappa_{n}\right) x_{n}^{3}=x_{n}+\kappa_{n} x_{n}^{3}, \\
2\left(x_{n}+\kappa_{n} x_{n}^{3}\right) f_{n}\left(\left(x_{l}\right)_{0 \leq l \leq n}\right)+f_{n}^{2}\left(\left(x_{l}\right)_{0 \leq l \leq n}\right) \leq 2\left|x_{n}+\kappa_{n} x_{n}^{3}\right| \sqrt{\delta_{n}^{(3)}}\left|x_{n}^{3}\right|+\delta_{n}^{(3)} x_{n}^{6} \\
\leq 2 \sqrt{\delta_{n}^{(3)}} x_{n}^{4}+\left(\delta_{n}^{(3)}+2 \kappa_{n} \sqrt{\delta_{n}^{(3)}}\right) x_{n}^{6} .
\end{gathered}
$$


Then, after returning to (3.8), we have

$$
\begin{aligned}
F_{n+1}^{2}\left(x_{n+1}\right) \leq & F_{n}^{2}\left(x_{n}\right)-2\left(a_{n}-\kappa_{n}\right) F_{n}\left(x_{n}\right) x_{n}^{3}+\left(a_{n}-\kappa_{n}\right)^{2} x_{n}^{6} \\
& +2 f_{n}\left(\left(x_{l}\right)_{0 \leq l \leq n}\right)\left(x_{n}+\kappa_{n} x_{n}^{3}\right)+f_{n}^{2}\left(\left(x_{l}\right)_{0 \leq l \leq n}\right) \\
& +\sigma_{n}^{2}\left(\left(x_{l}\right)_{0 \leq l \leq n}\right) \eta_{n+1}+\Delta m_{n+1}^{(1)} \\
\leq & F_{n}^{2}\left(x_{n}\right)-2\left(a_{n}-\kappa_{n}\right)\left(x_{n}+a_{n} x_{n}^{3}\right) x_{n}^{3}+\left(a_{n}-\kappa_{n}\right)^{2} x_{n}^{6} \\
& +2 \sqrt{\delta_{n}^{(3)}} x_{n}^{4}+\left(\delta_{n}^{(3)}+2 \kappa_{n} \sqrt{\delta_{n}^{(3)}}\right) x_{n}^{6}+\lambda_{n} \eta_{n+1}\left(1+x_{n}^{6}\right) \\
& +\delta_{n}^{(1)} \eta_{n+1} x_{n}^{4}+\delta_{n}^{(2)} \eta_{n+1} x_{n}^{6}+\Delta m_{n+1}^{(1)} \\
= & F_{n}^{2}\left(x_{n}\right)+\lambda_{n} \eta_{n+1}-\left[2\left(a_{n}-\kappa_{n}\right)-2 \sqrt{\delta_{n}^{(3)}}-\delta_{n}^{(1)} \eta_{n+1}\right] x_{n}^{4} \\
& -\left[\left(a_{n}^{2}-\kappa_{n}^{2}\right)-\delta_{n}^{(3)}-2 \kappa_{n} \sqrt{\delta_{n}^{(3)}}-\delta_{n}^{(2)} \eta_{n+1}-\lambda_{n} \eta_{n+1}\right] x_{n}^{6}+\Delta m_{n+1}^{(1)} .
\end{aligned}
$$

Now, recall that $\delta_{n}^{(j)}, j=1,2,3$, are supposed to be small enough such that conditions (3.4) and (3.5) with real constants $N_{1} \geq 0, \varepsilon_{1} \geq 0$, and $\varepsilon_{2} \geq 0$ satisfying $\varepsilon_{1}+\varepsilon_{2}>0$ hold for all $n \geq N_{1}$. Without loss of generality, we may suppose that $N_{1}=0$ (otherwise, we can start with summing up from $N_{1}$ onwards below). Through telescoping and estimation of the quadratic differences $F_{k}^{2}\left(x_{k}\right)-F_{k-1}^{2}\left(x_{k-1}\right)$ by (3.11), we obtain

$$
F_{n}^{2}\left(x_{n}\right)=\sum_{k=1}^{n}\left(F_{k}^{2}\left(x_{k}\right)-F_{k-1}^{2}\left(x_{k-1}\right)\right)+F_{0}^{2}\left(x_{0}\right) \leq F_{0}^{2}\left(x_{0}\right)+A_{n}^{1}-A_{n}^{2}+m_{n},
$$

where

$$
A_{n}^{1}=\sum_{i=1}^{n-1} \lambda_{i} \eta_{i+1}, \quad A_{n}^{2}=\sum_{i=1}^{n-1}\left(\varepsilon_{1} x_{n}^{4}+\varepsilon_{2} x_{n}^{6}\right)
$$

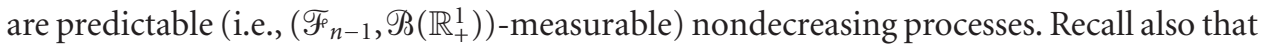
condition (3.2) guarantees that $\lim _{n \rightarrow+\infty} A_{n}^{1}$ exists and is finite. Define $Z_{n}=F_{n}^{2}\left(x_{n}\right)$ along the sequence of $x_{n}$. Then Lemma 2.2 can be applied to $Z=\left\{Z_{n}\right\}_{n \in \mathbb{N}}$, and hence the limit $Z_{+\infty}:=\lim _{n \rightarrow+\infty} F_{n}^{2}\left(x_{n}\right)$ a.s. exists and is finite too. Thus, we also know this fact about $\lim \sup _{n \rightarrow+\infty} F_{n}^{2}\left(x_{n}\right)$ which equals $Z_{+\infty}$. Note that, by squeezing theorem from calculus, we have

$$
0 \leq \limsup _{n \rightarrow+\infty} x_{n}^{2} \leq \limsup _{n \rightarrow+\infty}\left[x_{n}^{2}+\left(\inf _{n \in \mathbb{N}} a_{n}\right)^{2} x_{n}^{6}\right] \leq \limsup _{n \rightarrow+\infty} F_{n}^{2}\left(x_{n}\right)<+\infty .
$$

Therefore, the limit $\limsup _{n \rightarrow+\infty} x_{n}^{2}$ is finite (a.s.). In the constant case $a_{n}=a$ (a constant), we can obtain the same conclusion for the $\operatorname{limit}_{n \rightarrow+\infty} x_{n}^{2}$ instead of limsup using the unique invertibility of the function $F$ with parameter $a$ and the continuity of its 
inverse $F^{-1}$ by Lemma 2.3. Thus, we may conclude that the finite limit $\limsup _{n \rightarrow \infty} x_{n}^{2}=$ $c_{0}^{2}(\omega)$ a.s. exists, where $c_{0}^{2}(\omega) \geq 0$. It remains to prove that $\lim _{n \rightarrow+\infty} x_{n}^{2}=0$. Suppose, indirectly, that the opposite is true. Then there exists a.s. a finite $c_{0}^{2}(\omega)>0$ on $\Omega_{1}=\{\omega$ : $\left.\limsup _{n \rightarrow+\infty} x_{n}^{2}(\omega)=c_{0}^{2}(\omega)>0\right\}$ with $\mathbb{P}\left(\Omega_{1}\right)=p_{1}>0$. There also exist a subsequence $\left\{x_{n_{k}}\right\}_{n_{k} \in \mathbb{N}}$ and an integer $N(\omega)$ such that

$$
\limsup _{n_{k} \rightarrow+\infty} x_{n_{k}}^{2}=c_{0}^{2}(\omega), \quad \frac{3 c_{0}^{2}}{2}(\omega) \geq x_{n_{k}}^{2}(\omega) \geq \frac{c_{0}^{2}}{2}(\omega)
$$

for all $n_{k} \geq N(\omega)$ on $\omega \in \Omega_{1}$. Let $I_{N}^{n}=\left\{n_{k} \in \mathbb{N}: n \geq n_{k} \geq N\right.$, (3.15) holds $\}$. Note that the cardinality $\#\left(I_{N}^{n}\right)$ tends to $+\infty$ as $n \rightarrow+\infty$. Then, for all $\omega \in \Omega_{1}$, some a.s. finite $c_{1}^{2}(\omega)=$ $\left(\varepsilon_{1}\left(\left(c_{0} / 2\right)(\omega)\right)^{2}+\varepsilon_{2}\left(\left(c_{0} / 2\right)(\omega)\right)^{3}\right)>0$ and for all $n>N(\omega)$, we have

$$
\begin{aligned}
A_{n}^{2}(\omega) & =\sum_{i=1}^{n}\left(\varepsilon_{1} x_{i}^{4}+\varepsilon_{2} x_{i}^{6}\right)=\sum_{i=1}^{N}\left(\varepsilon_{1} x_{i}^{4}+\varepsilon_{2} x_{i}^{6}\right)+\sum_{i=N}^{n}\left(\varepsilon_{1} x_{i}^{4}+\varepsilon_{2} x_{i}^{6}\right) \\
& \geq \sum_{i=N}^{n}\left(\varepsilon_{1} x_{i}^{4}+\varepsilon_{2} x_{i}^{6}\right) \geq \sum_{i=N, i \in I_{N}^{n}}^{n}\left(\varepsilon_{1}\left(\frac{c_{0}^{2}}{2}(\omega)\right)^{2}+\varepsilon_{2}\left(\frac{c_{0}^{2}}{2}(\omega)\right)^{3}\right) \\
& =\sum_{i=N, i \in I_{N}^{n}}^{n} c_{1}^{2}(\omega)=\#\left(I_{N}^{n}\right) c_{1}^{2}(\omega) \longrightarrow+\infty,
\end{aligned}
$$

as $n \rightarrow+\infty$. Therefore, $\limsup _{n \rightarrow+\infty} A_{n}^{2}=\lim _{n \rightarrow+\infty} A_{n}^{2}=+\infty$. This result contradicts the finiteness of $\lim _{n \rightarrow+\infty} A_{n}^{2}$ resulting from Lemma 2.2. Thus, Theorem 3.1 is proved.

Remarks 3.2. We briefly discuss the conditions of Theorem 3.1.

(i) We can suppose that $\delta_{n}^{(j)}, j=1,2$, are just arbitrary nonrandom constants with sufficiently small $\delta^{(3)}$, but in this case, to ensure the fulfilment of (3.2), (3.3), (3.4), and (3.5), we need to require that $\eta_{n} \rightarrow 0$ as $n \rightarrow+\infty$.

(ii) If $\eta_{n}$ does not tend to 0 , then $\lambda_{n}$ has to tend to 0 . In this case, $\lambda_{n}$ can be considered as a small enough number (in (3.5)), and instead of (3.2), we can demand

$$
\left|\sigma_{n}\left(\left(x_{l}\right)_{0 \leq l \leq n}\right)\right|^{2} \leq \lambda_{n}+\delta_{n}^{(1)} x_{n}^{4}+\delta_{n}^{(2)} x_{n}^{6}, \quad \sum_{n=1}^{+\infty} \lambda_{n} \eta_{n}<+\infty
$$

Then a similar analysis as in the proof before leads to the less-restrictive condition

$$
\left(a_{n}^{2}-\kappa_{n}^{2}\right)-\delta_{n}^{(2)} \eta_{n+1}-\delta_{n}^{(3)}-2 \kappa_{n} \sqrt{\delta_{n}^{(3)}} \geq \varepsilon_{2}
$$

which replaces condition (3.5), and hence asymptotic stability can be established. Consequently, Theorem 3.1 is valid under the hypotheses of (3.1), (3.17), (3.3), (3.4), and (3.18) too.

(iii) If $\eta_{n} \rightarrow 0$ is fast enough as $n \rightarrow+\infty$ (e.g., when $\sum_{n=1}^{+\infty} \eta_{n}<+\infty$ ), then $\lambda_{n}$ can be even bounded below away from zero. In this case, it is reasonable that, instead of (3.2), 
we require

$$
\left|\sigma_{n}\left(\left(x_{l}\right)_{0 \leq l \leq n}\right)\right|^{2} \leq \lambda_{n}\left(1+x_{n}^{6}\right)+\delta_{n}^{(1)} x_{n}^{4}, \quad \sum_{n=1}^{+\infty} \lambda_{n} \eta_{n}<+\infty .
$$

That means that, by putting $\delta_{n}^{(2)}=0$, Theorem 3.1 with conditions (3.1), (3.19), (3.3), (3.4), and (3.5) is applicable and implies asymptotic stability of (1.1) as well.

\section{An application to numerical methods for CSDEs}

As an example of applicability of our main result, consider the Itô-interpreted CSDEs

$$
d X_{t}=\left(\rho \sin \left(\left[X_{t}\right]^{3}\right)-\gamma^{2}\left[X_{t}\right]^{3}\right) d t+\left(\sigma_{0} \frac{\left|X_{t}\right|^{3}}{1+t}+\sigma_{1}\left[X_{t}\right]^{2}+\sigma_{2}\left[X_{t}\right]^{3}\right) d W_{t}
$$

with real constants $\rho, \gamma, \sigma_{0}, \sigma_{1}$, and $\sigma_{2}$, discretized by the partially drift-implicit $\theta$-method

$$
\begin{aligned}
x_{n+1}= & x_{n}+\left(\rho \sin \left(\left[x_{n}\right]^{3}\right)-\gamma^{2}\left(\theta_{n}\left[x_{n+1}\right]^{3}+\left(1-\theta_{n}\right)\left[x_{n}\right]^{3}\right)\right) \Delta_{n} \\
& +\left(\sigma_{0} \frac{\left|x_{n}\right|^{3}}{1+t_{n}}+\sigma_{1}\left[x_{n}\right]^{2}+\sigma_{2}\left[x_{n}\right]^{3}\right) \xi_{n+1}
\end{aligned}
$$

where $\xi_{n+1}=\Delta W_{n}$ with $\eta_{n+1}=\mathbb{E}\left[\xi_{n+1}\right]^{2}=\Delta_{n}$, while using nonrandom step sizes $\Delta_{n}$. Obviously, both equations possess the trivial equilibrium 0. For the CSDE (4.1), 0 is a locally stable equilibrium (a.s.). This fact can be seen immediately from a discussion of the stability of a related linearized equation $d X_{t} / d t=0$ for $X$ (linearized about its steady state $0)$. A discussion with respect to global a.s. asymptotic stability and instability of CSDE (4.1) is more delicate. One obvious result in this direction is given as follows.

Theorem 4.1. Assume that the initial values $X_{0}=x_{0}$ are independent of the $\sigma$-algebra $\sigma\left(W_{s}: s \geq 0\right), \sigma_{0}^{2}+\sigma_{2}^{2}=0$ and $2\left(\gamma^{2}-|\rho|\right)-\sigma_{1}^{2}>0$. Then the trivial solution 0 of CSDE (4.1) is globally asymptotically stable (a.s.).

Proof. Apply Itô formula to the Lyapunov function $V(x)=x^{2}$ for the solution of (4.1). Thus, combining with the fact that $|\sin (z) / z| \leq 1$ for all $z \in \mathbb{R}$, we obtain that, for $t \geq 0$,

$$
\begin{aligned}
d X_{t}^{2} & =\left(2 \rho \sin \left(\left[X_{t}\right]^{3}\right) X_{t}-2 \gamma^{2}\left[X_{t}\right]^{4}+\sigma_{1}^{2}\left[X_{t}\right]^{4}\right) d t+d m_{t} \\
& \leq\left(2\left(|\rho|-\gamma^{2}\right)+\sigma_{1}^{2}\right)\left[X_{t}\right]^{4} d t+d m_{t},
\end{aligned}
$$

where $m=\left\{m_{t}\right\}_{t \geq 0}$, with $m_{t}=2 \sigma_{1} \int_{0}^{t}\left[X_{s}\right]^{3} d W_{s}$, is a locally square-integrable martingale. Suppose that $\mu:=2\left(\gamma^{2}-|\rho|\right)-\sigma_{1}^{2}>0$. Hence, the asymptotic behavior of the nonnegative 
semimartingale $X_{t}^{2}$ governed by (4.3) is controlled by the solution $Z=\left\{Z_{t}\right\}_{t \geq 0}$ of

$$
d Z_{t}=-\mu\left[Z_{t}\right]^{2} d t+d m_{t},
$$

with sufficiently large $\mu=2\left(\gamma^{2}-|\rho|\right)-\sigma_{1}^{2}>0$. Therefore, we may decompose its drift into nondecreasing processes $A^{1}=\left\{A_{t}^{1}\right\}_{t \geq 0}$ and $A^{2}=\left\{A_{t}^{2}\right\}_{t \geq 0}$ given by

$$
A_{t}^{1}=0, \quad A_{t}^{2}=\mu \int_{0}^{t}\left[Z_{s}\right]^{2} d s
$$

Notice also that $Z=\left\{Z_{t}\right\}_{t \geq 0}$ is a nonnegative supermartingale. Now, we may apply Doob's martingale convergence theorems or a continuous version of Lemma 2.2 (which is also found in [2, Chapter 2, Theorem 7, page 139] and generalized in [3]) in order to know about the existence of the finite limits $Z_{+\infty}=\lim _{t \rightarrow+\infty} X_{t}^{2}$ and $\lim _{t \rightarrow+\infty} A_{t}^{2}<+\infty$. It remains to show that $X_{t}$ converges to 0 (a.s.). Note that $Z_{t} \geq 0$ for all $t \geq 0, \mu>0$ and $\lim _{t \rightarrow+\infty} A_{t}^{2}=\mu \int_{0}^{+\infty}\left[Z_{s}\right]^{2} d s<+\infty$ holds. It is well known that the convergence of positive integrand $\left[Z_{s}\right]^{2}$ to 0 as $s$ tends to $+\infty$ is necessary for the convergence of the improper integral in $\lim _{t \rightarrow+\infty} A_{t}^{2}$. Hence, $\lim _{t \rightarrow+\infty} Z_{t}^{2}=0$ (a.s.) implies that $\lim _{t \rightarrow+\infty} X_{t}^{2}=0$ and $X_{+\infty}=\lim _{t \rightarrow+\infty} X_{t}=0$ (a.s.). Therefore, the proof is complete.

However, for DSDE (4.2), the situation might depend on the choice of step sizes $\Delta_{n}$.

Corollary 4.2. Let $x=\left\{x_{n}\right\}_{n \in \mathbb{N}}$ satisfy the stochastic difference equation (4.2) under the above-mentioned conditions with $\gamma^{2}>0, \theta_{n}>0.5$, and nonrandom variable step sizes $\Delta_{n}$ which are uniformly bounded such that

$$
\exists \Delta_{a}, \Delta_{b}: \forall n \in \mathbb{N}, \quad 0<\Delta_{b} \leq \Delta_{n} \leq \Delta_{a}<+\infty,
$$

and $\left\{\theta_{n} \Delta_{n}\right\}_{n \in \mathbb{N}}$ is nonincreasing. Furthermore, assume that

$$
\begin{gathered}
c_{1}=2\left(\gamma^{2}-|\rho|\right)-3 \sigma_{1}^{2} \geq 0 \\
c_{2}=\inf _{n \in \mathbb{N}}\left(\left(\gamma^{4}\left(2 \theta_{n}-1\right)+2 \gamma^{2}\left(1-\theta_{n}\right)|\rho|-\rho^{2}\right) \Delta_{n}-3 \sigma_{2}^{2}-\frac{3 \sigma_{0}^{2}}{\left(1+t_{n}\right)^{2}}\right) \geq 0 .
\end{gathered}
$$

Then the limits $\liminf _{n \rightarrow+\infty} x_{n}^{2}, \limsup _{n \rightarrow+\infty} x_{n}^{2}, \liminf _{n \rightarrow+\infty} x_{n}$, and $\limsup _{n \rightarrow+\infty} x_{n}$ for the sequences $x=\left\{x_{n}\right\}_{n \in \mathbb{N}}$ governed by equation (4.2) are finite (i.e., independent of the magnitude of its initial values $x_{0}$ ). Moreover, if additionally $c_{1}+c_{2}>0$, then the related difference equation (4.2) possesses an a.s. globally asymptotically stable trivial solution.

Proof. Apply Theorem 3.1. For this purpose, note that equation (4.2) has the form (1.1) with $f_{n}\left(\left(x_{l}\right)_{0 \leq l \leq n}\right)=\rho \sin \left(x_{n}^{3}\right) \Delta_{n}, a_{n}=\gamma^{2} \theta_{n} \Delta_{n}>0$, and $\kappa_{n}=-\gamma^{2}\left(1-\theta_{n}\right) \Delta_{n}$. It remains to check conditions (3.1), (3.2), (3.3), (3.4), and (3.5). After division by $\gamma^{2} \Delta_{n}$, condition (3.1) is equivalent to $\theta_{n}>\left|1-\theta_{n}\right|$ which is trivially guaranteed by the choice $\theta_{n}>0.5$. Furthermore, define

$$
\lambda_{n}=\frac{3 \sigma_{0}^{2}}{\left(1+t_{n}\right)^{2}}, \quad \delta_{n}^{(1)}=3 \sigma_{1}^{2}, \quad \delta_{n}^{(2)}=3 \sigma_{2}^{2}, \quad \delta_{n}^{(3)}=\rho^{2} \Delta_{n}^{2} .
$$


One easily estimates

$$
\begin{aligned}
\left|\sigma_{n}\left(\left(x_{l}\right)_{0 \leq l \leq n}\right)\right|^{2} & =\left|\sigma_{0} \frac{\left|x_{n}\right|^{3}}{1+t_{n}}+\sigma_{1}\left[x_{n}\right]^{2}+\sigma_{2}\left[x_{n}\right]^{3}\right|^{2} \\
& \leq 3\left(\sigma_{0}^{2} \frac{\left|x_{n}\right|^{6}}{\left(1+t_{n}\right)^{2}}+\sigma_{1}^{2}\left[x_{n}\right]^{4}+\sigma_{2}^{2}\left[x_{n}\right]^{6}\right) \\
& \leq \lambda_{n}\left(1+x_{n}^{6}\right)+\delta_{n}^{(1)} x_{n}^{4}+\delta_{n}^{(2)} x_{n}^{6}, \\
\sum_{n=1}^{+\infty} \lambda_{n} \mathbb{E}\left[\xi_{n+1}^{2}\right] & =\sum_{n=1}^{+\infty} \frac{3 \sigma_{0}^{2}}{\left(1+t_{n}\right)^{2}} \Delta_{n} \\
& \leq 3 \sigma_{0}^{2} \Delta_{a} \sum_{n=1}^{+\infty} \frac{1}{\left(1+n \Delta_{b}\right)^{2}}<3 \sigma_{0}^{2} \frac{\Delta_{a}}{\left(\Delta_{b}\right)^{2}} \frac{\pi^{2}}{6}<+\infty, \\
\left|f_{n}\left(\left(x_{l}\right)_{0 \leq l \leq n}\right)\right|^{2} & =\left|\rho \sin \left(x_{n}^{3}\right) \Delta_{n}\right|^{2} \leq \delta_{n}^{(3)} x_{n}^{6},
\end{aligned}
$$

hence conditions (3.2) and (3.3) are fulfilled too. It remains to check (3.4) and (3.5). To evaluate condition (3.4), we compute

$$
\begin{aligned}
2\left(a_{n}-\kappa_{n}\right)-2 \sqrt{\delta_{n}^{(3)}}-\delta_{n}^{(1)} \eta_{n+1} & =2\left(\gamma^{2} \theta_{n} \Delta_{n}+\gamma^{2}\left(1-\theta_{n}\right) \Delta_{n}\right)-2|\rho| \Delta_{n}-3 \sigma_{1}^{2} \Delta_{n} \\
& =\left(2\left(\gamma^{2}-|\rho|\right)-3 \sigma_{1}^{2}\right) \Delta_{n} \geq\left(2\left(\gamma^{2}-|\rho|\right)-3 \sigma_{1}^{2}\right) \Delta_{b} \\
& =c_{1} \Delta_{b}=: \varepsilon_{1} .
\end{aligned}
$$

Condition (3.5) is verified by

$$
\begin{aligned}
\left(a_{n}^{2}-\right. & \left.\kappa_{n}^{2}\right)-\delta_{n}^{(2)} \eta_{n+1}-\delta_{n}^{(3)}-2 \kappa_{n} \sqrt{\delta_{n}^{(3)}}-\lambda_{n} \eta_{n+1} \\
& =\gamma^{4} \theta_{n}^{2} \Delta_{n}^{2}-\gamma^{4}\left(1-\theta_{n}\right)^{2} \Delta_{n}^{2}-3 \sigma_{2}^{2} \Delta_{n}-\rho^{2} \Delta_{n}^{2}+2 \gamma^{2}\left(1-\theta_{n}\right) \Delta_{n}^{2}|\rho|-\frac{3 \sigma_{0}^{2}}{\left(1+t_{n}\right)^{2}} \Delta_{n} \\
& =\left(\gamma^{4}\left(2 \theta_{n}-1\right) \Delta_{n}-3 \sigma_{2}^{2}-\rho^{2} \Delta_{n}+2 \gamma^{2}\left(1-\theta_{n}\right) \Delta_{n}|\rho|-\frac{3 \sigma_{0}^{2}}{\left(1+t_{n}\right)^{2}}\right) \Delta_{n} \\
& \geq \inf _{n \in \mathbb{N}}\left(\left(\gamma^{2}\left[\gamma^{2}\left(2 \theta_{n}-1\right)+2\left(1-\theta_{n}\right)|\rho|\right]-\rho^{2}\right) \Delta_{n}-3 \sigma_{2}^{2}-\frac{3 \sigma_{0}^{2}}{\left(1+t_{n}\right)^{2}}\right) \Delta_{b}=c_{2} \Delta_{b} .
\end{aligned}
$$

Therefore, we have found mathematical expressions for $\varepsilon_{1}=c_{1} \Delta_{b} \geq 0$ and $\varepsilon_{2}=c_{2} \Delta_{b} \geq 0$ under (4.7) and (4.8). Summarizing our prior calculations, the validity of (3.1), (3.2), (3.3), (3.4), and (3.5) could be verified when $c_{1}+c_{2}>0$. Hence, Theorem 3.1 can be applied directly. If $c_{1}=c_{2}=0$, then the limits liminf and limsup are finite by the application of Lemmas 2.1, 2.2, and 2.3 as in the proof of Theorem 3.1. Hence, the proof of Corollary 4.2 is complete. 
Consequently, under (4.6), (4.7), and (4.8), our main result says that 0 is an asymptotically stable equilibrium for the method (4.2) with probability one. Thus, this extends results for the linear case (cf. $[9,11,13])$ to the nonlinear case with cubic main drift part.

A special role of the magnitudes of $\sigma_{i}(i=0,1,2)$ is seen for the stability of the dynamics of equations (4.2). If $\Delta_{a}$ tends to 0 and all other parameters are fixed with $\sigma_{0}^{2}+\sigma_{2}^{2}>0$, then (4.8) is violated. This fact is natural since $\omega$-dependent explosions of strong solutions of (4.1) as limits of (4.2) might occur due to the interaction of its inherent nonlinearities. If $\rho$ and $\sigma_{1}$ are small enough (i.e., also $\gamma^{2}>0$ is large enough), then condition (4.7) can be fulfilled. It also confirms the fact that conditions on the magnitudes of $\rho, \gamma$, and $\sigma_{i}$ must play an essential role in the proof of strong existence and uniqueness results for the nonlinear equations (4.1) based on discrete approximation techniques.

In view of Theorem 4.1, a refinement of Corollary 4.3 with slightly relaxed conditions is found as follows.

Corollary 4.3. Let $x=\left\{x_{n}\right\}_{n \in \mathbb{N}}$ satisfy the stochastic difference equation (4.2) with $\gamma^{2}>0$, $\theta_{n}>0.5, \sigma_{0}^{2}+\sigma_{2}^{2}=0$, and nonrandom variable step sizes $\Delta_{n}$ which are uniformly bounded such that there exist $\Delta_{a}, \Delta_{b}$ such that for all $n \in \mathbb{N}, 0<\Delta_{b} \leq \Delta_{n} \leq \Delta_{a}<+\infty$, and $\left\{\theta_{n} \Delta_{n}\right\}_{n \in \mathbb{N}}$ is nonincreasing. Furthermore, assume that

$$
\begin{gathered}
c_{1}=2\left(\gamma^{2}-|\rho|\right)-\sigma_{1}^{2} \geq 0, \\
c_{2}=\inf _{n \in \mathbb{N}}\left(\gamma^{4}\left(2 \theta_{n}-1\right)+2 \gamma^{2}\left(1-\theta_{n}\right)|\rho|-\rho^{2}\right) \geq 0 .
\end{gathered}
$$

Then, the limits $\liminf \operatorname{in}_{n \rightarrow+\infty} x_{n}^{2}$, $\limsup _{n \rightarrow+\infty} x_{n}^{2}, \liminf _{n \rightarrow+\infty} x_{n}$, and $\limsup _{n \rightarrow+\infty} x_{n}$ for the sequences $x=\left\{x_{n}\right\}_{n \in \mathbb{N}}$ governed by equation (4.2) are finite (i.e., independent of the magnitude of its initial values $x_{0}$ ). Moreover, if additionally $c_{1}+c_{2}>0$, then the related difference equation (4.2) possesses an a.s. globally asymptotically stable trivial solution.

Proof. Apply Theorem 3.1 as before. One can take $\lambda_{n}=0, \delta_{n}^{(1)}=\sigma_{1}^{2}, \delta_{n}^{(2)}=0$, and $\delta_{n}^{(3)}$. Then the only difference to the proof before is that we do not need to apply the discrete Hölder inequality in the estimation of $\left|\sigma_{n}\left(\left(x_{l}\right)_{0 \leq l \leq n}\right)\right|^{2}$ here. Furthermore, trivially, $\sum_{n=1}^{+\infty} \lambda_{n} \eta_{n}=0$ holds. Thus, the proof is obvious.

Remark 4.4. Conditions (4.13) and (4.14) do not depend on the choice of step sizes $\Delta_{n}$. This fact is due to the specific construction of partially drift-implicit $\theta$-methods with parameters $\theta_{n}>0.5$ only under the right choice of noise (i.e., when $\sigma_{0}=0$ and $\sigma_{2}=0$ ). Moreover, condition (4.13) coincides with that of Theorem 4.1 resulting from the behavior of solutions of the underlying continuous equation (4.1). Condition (4.14) exhibits the interactive interplay of the choice of parameters $\theta_{n}$, the nonlinearity intensity $\gamma^{2}$, and the rotation-controlling magnitude of $\rho$. It shows that both noise and rotation terms have to be chosen carefully in order not to destabilize the long-term dynamics by partially drift-implicit $\theta$-methods (4.2). Anyway, note that we have only found sufficient conditions for asymptotic stability. Hence, necessary and sufficient conditions may still depend on the choice of step sizes $\Delta_{n}$.

Further remarks. More care is needed when choosing variable step size algorithms in order to achieve adequate convergence and asymptotic stability results. An analysis in this 
direction is omitted here. In passing, we note that conditions (4.6) are not always fullfilled in the case of variable step sizes $\Delta_{n}$; for example, $\Delta_{n}=1 / n$ which are not very meaningful anyway since they can run below any natural machine accuracy (note that in this case we cannot clearly distinguish between computer-generated noise and the random noise originating from the underlying analytic equations). Again, we have some indications that the choice of step sizes following the restriction (4.6) gives meaningful qualitative results for stochastic numerical methods (cf. $[9,12,13])$. These latter remarks might be interesting for the implementation and convergence proofs referring to the use of variable step sizes in stochastic numerical algorithms instead of the more trivial case of constant ones. It is worth noting that our main result (Theorem 3.1) is applicable to both "weak" and "strong" approximations of CSDEs (1.2) with variable (but nonrandom) step sizes since we have only exploited techniques from martingale theory. In particular, our proofs rely heavily on the assumption that $\left\{\xi_{n}\right\}_{n \in \mathbb{N}}$ are martingale differences, which is the case for "weak" and "strong" approximations of CSDEs.

Our results can be extended to the case of stochastic Volterra-type difference equations while using the method of Lyapunov-Krasovskii functionals as similarly done in $[5,6]$, a subject that will be discussed in our future works. We do not claim that our results are of the most general nature. However, we have shown their verification and potential applicability.

\section{Acknowledgments}

The authors are grateful to Dr. John A. D. Appleby for a very interesting discussion in Atlanta in May 2003. We were particularly inspired to write up this paper by the works of $[4,7,8,11]$. Moreover, all comments of the anonymous referees were greatly appreciated by the authors.

\section{References}

[1] J. T. Edwards, N. J. Ford, J. A. Roberts, and L. Shaikhet, Stability of a discrete nonlinear integrodifferential equation of convolution type, Stab. Control Theory Appl. 3 (2000), no. 1, 24-37.

[2] R. Sh. Liptser and A. N. Shiryayev, Theory of Martingales, Mathematics and Its Applications (Soviet Series), vol. 49, Kluwer Academic Publishers, Dordrecht, 1989.

[3] A. V. Mel'nikov and A. E. Rodkina, Martingale approach to the procedures of stochastic approximation, Proceedings of the Third Finnish-Soviet Symposium on Probability Theory and Mathematical Statistics (Turku, Finland, 1991) (H. Niemi et al., eds.), Front. Pure Appl. Probab., vol. 1, VSP, Utrecht, 1993, pp. 165-182.

[4] V. R. Nosov, Stability of cubic equation with delay, Abstracts of International Conference "Functional Differential Equations and Applications" (Beer-Sheva, Israel, 2002), College of Judea \& Samaria, Research Institute, Ariel, 2003, pp. 48-49.

[5] A. Rodkina and X. Mao, On boundedness and stability of solutions of nonlinear difference equation with nonmartingale type noise, J. Differ. Equations Appl. 7 (2001), no. 4, 529-550.

[6] A. Rodkina, X. Mao, and V. Kolmanovskii, On asymptotic behaviour of solutions of stochastic difference equations with Volterra type main term, Stochastic Anal. Appl. 18 (2000), no. 5, $837-857$.

[7] A. Rodkina and V. Nosov, On stability of stochastic delay cubic equations, Proceedings of Dynamic Systems and Applications (Atlanta, 2003) (M. Sambandham, ed.), vol. 4, Dynamic Publisher, Georgia, in press. 
[8] L. B. Ryashko and H. Schurz, Mean square stability analysis of some linear stochastic systems, Dynam. Systems Appl. 6 (1997), no. 2, 165-189.

[9] H. Schurz, Stability, Stationarity, and Boundedness of Some Implicit Numerical Methods for Stochastic Differential Equations and Applications, Logos Verlag, Berlin, 1997.

[10] , Partial and linear-implicit numerical methods for nonlinear SDEs, unpublished manuscript, Universidad de Los Andes, Bogota, 1998.

[11] The invariance of asymptotic laws of linear stochastic systems under discretization, ZAMM Z. Angew. Math. Mech. 79 (1999), no. 6, 375-382.

[12] Moment attractivity, stability and contractivity exponents of stochastic dynamical systems, Discrete Contin. Dynam. Systems 7 (2001), no. 3, 487-515.

[13] _ Numerical analysis of stochastic differential equations without tears, Handbook of Stochastic Analysis and Applications (D. Kannan and V. Lakshmikantham, eds.), Statist. Textbooks Monogr., vol. 163, Dekker, New York, 2002, pp. 237-359.

[14] A. N. Shiryaev, Probability, Graduate Texts in Mathematics, vol. 95, Springer-Verlag, New York, 1996.

[15] P. Spreij, Recursive approximate maximum likelihood estimation for a class of counting process models, J. Multivariate Anal. 39 (1991), no. 2, 236-245.

Alexandra Rodkina: Department of Mathematics and Computer Science, University of the West Indies at Mona, Kingston 7, Jamaica

E-mail address: alexandra.rodkina@uwimona.edu.jm

Henri Schurz: Department of Mathematics, Southern Illinois University, 1245 Lincoln Drive, Carbondale, IL 62901-4408, USA

E-mail address: hschurz@math.siu.edu 